\title{
Calendar effects to forecast influenza seasonality: A case study in Milwaukee, WI
}

\section{R. B. Simpson ${ }^{1}$, Aishwarya Venkat ${ }^{1}$, Tania Alarcon ${ }^{1}$, Ken Chui ${ }^{1}$, Yuri Naumov ${ }^{2}$, Jack Gorski ${ }^{2}$, Sanjib Bhattacharyya ${ }^{3}$, Elena Naumova ${ }^{1}$}

${ }^{1}$ Friedman School of Nutrition Science and Policy, Tufts University, Malden, Massachusetts, United States, ${ }^{2}$ Blood Research Center, Milwaukee, Wisconsin, United States, ${ }^{3}$ MHDL, Milwaukee, Wisconsin, United States

\section{Objective}

In the presented study, we examined the impact of school holidays (Autumn, Winter, Summer, and Spring Breaks) and social events (Super Bowl, NBA Finals, World Series, and Black Friday) for five age groups (<4, 5-24, 25-44, 45-64, >65 years) on four health outcomes of influenza (total tested, all influenza positives, positives for influenza A, and B) in Milwaukee, WI, in 2004-2009 using routine surveillance.

\section{Introduction}

Influenza viral infection is contentious, has a short incubation period, yet preventable if multiple barriers are employed. At some extend school holidays and travel restrictions serve as a socially accepted control measure [1,2]. A study of a spatiotemporal spread of influenza among school-aged children in Belgium illustrated that changes in mixing patterns are responsible for altering disease seasonality [3]. Stochastic numerical simulations suggested that weekends and holidays can delay disease seasonal peaks, mitigate the spread of infection, and slow down the epidemic by periodically dampening transmission. While Christmas holidays had the largest impact on transmission, other school breaks may also help in reducing an epidemic size. Contrary to events reducing social mixing, sporting events and mass gatherings facilitate the spread of infections [4]. A study on county-level vital statistics of the US from 1974-2009 showed that Super Bowl social mixing affects influenza dissemination by decreasing mortality rates in older adults in Bowl-participating counties. The effect is most pronounced for highly virulent influenza strains and when the Super Bowl occurs closer to the influenza seasonal peak. Simulation studies exploring how social mixing affects influenza spread [5] demonstrated that impact of the public gathering on prevalence of influenza depends on time proximity to epidemic peak. While the effects of holidays and social events on seasonal influenza have been explored in surveillance time series and agent-based modeling studies, the understanding of the differential effects across age groups is incomplete.

\section{Methods}

The City of Milwaukee Health Department Laboratory (MHDL), Wisconsin routinely collect tests from residents of metropolitan areas and vicinities of the Marquette University (MU). We obtained weekly counts of total tested, all influenza positives, positives for influenza A and B, from MHDL between 5/16/04- 3/7/09 (before the surge of tests associated with "swine flu"). Cases for $<1$ and 1-4 age groups were combined. Meteorological data are routinely collected by a monitoring station at the General Mitchell International airport located 7.5 miles from Milwaukee. Daily dewpoint values representing the perceived ambient temperature corrected for the air moisture content were downloaded from the open source website [6] and aggregated to weekly averages with Sunday designating the beginning of each week. School holidays were obtained from academic calendars on the MU website with holiday weeks defined as having one or more school holiday observed [7]. Selected social events were retrieved from a public website [8]. As part of exploratory analysis, average cases per week (c/w) for each outcome for school holiday and non-holiday weeks were compared using a non-parametric the Mann-Whitney U-test. We analyzed the association between weekly cases and holiday effects using negative binomial regression with sets of indicator variables for non-overlapping school holidays and social events and with adjustments for weather fluctuations with harmonic terms (Model 1). Results are presented as Relative Risk (RR) estimates along with their confidence intervals $(95 \% \mathrm{CI})$. Further analyses examined seasonal signatures (lead-lag structures) using a segmented regression approach for weekly counts and rates 5 academic weeks (aw) before, 2-6 weeks during, and 5 weeks after select holidays (Model 2). 


\section{Results}

Over 251 study weeks, 2282 tests were submitted, out of which 1098 cases were from 5-24 y.o. age group. 477 (21\%) tests we positive, with 399 (84\%) cases of influenza A (73 tests were not subtyped) and 78 (16\%) cases of influenza B. Figure 1 shows the time series of weekly counts of influenza tests and percent positives with superimposed information on school holiday occurrences. Overall, during 135 weeks of the school period the average number of tests was two times higher as compared to those during 116 holiday weeks $(11.9 \pm 10.3$ vs $5.8 \pm 6.5 \mathrm{c} / \mathrm{w}, \mathrm{p}<0.001)$. Similarly, the average weekly number of positive tests was higher in nonholiday than during holiday periods $(2.9 \pm 5.7$ vs $0.7 \pm 2.6 \mathrm{c} / \mathrm{w}, \mathrm{p}<0.001)$. The reduction in tests during holidays was confirmed by the regression model $(\mathrm{RR}=0.71 ; 95 \% \mathrm{CI}=[0.60-0.86])$. The reduction in weekly tests was most pronounced during the Winter Break $(15-19 \mathrm{aw})$ for all age groups $(4.8 \pm 3.0 \mathrm{c} / \mathrm{w}, \mathrm{p}<0.001 ; \mathrm{RR}=0.3 ; 95 \% \mathrm{CI}=[0.23-0.41])$ and especially for school-aged children, young adults and adults $(\mathrm{RR}=0.14 ; 95 \% \mathrm{CI}=[0.09-0.22]$ and $\mathrm{RR}=0.32 ; 95 \% \mathrm{CI}=[0.16-0.62]$ for 5-24 and 25-44 age groups, respectively). In contrast, during the Spring Break (27-30 aw) the number of tests has almost doubled $(20.4 \pm 10.4 \mathrm{c} / \mathrm{w} ; \mathrm{p}<0.001)$ as compared to the school period, with the most noticeable increase in 5-24 and 25-44 age groups. Spring Break differential effects were primarily due to later peaks in influenza B shown by segmented regression results in Figure 2. The seasonal increase in weekly rates is the steepest after the winter holidays. The effects of the selected sporting and social events were inconclusive.

\section{Conclusions}

The differential effects of calendar events on seasonal influenza can be detected by routine surveillance and further explored with respect to lead-lag structures. We recommend incorporating location-specific calendar effects in influenza near-term forecasting models tailored to susceptible age groups to better predict and assess targeted intervention measures.

\section{Acknowledgement}

NIH Grants: U19AI062627, NO1A150032.

\section{References}

1. Jackson C, Vynnycky E, Mangtani P. 2016. The relationship between school holidays and transmission of influenza in England and wales. Am J Epidemiol. 184(9), 644-51. PubMed https://doi.org/10.1093/aje/kww083

2. Chu Y, et al. 2017. Effects of school breaks on influenza-like illness incidence in a temperate Chinese region: an ecological study from 2008 to 2015. BMJ Open. 7(3), e013159. PubMed https://doi.org/10.1136/bmjopen2016-013159

3. Luca G, et al. 2018. The impact of regular school closure on seasonal influenza epidemics: a data-driven spatial transmission model for Belgium. BMC Infect Dis. 18(1), 29. PubMed https://doi.org/10.1186/s12879017-2934-3

4. Stoecker C, Sanders N, Barreca A. 2016. Success Is Something to Sneeze At: Influenza Mortality in Cities that Participate in the Super Bowl. Am J Health Econ. 2(1), 125-43. https://doi.org/10.1162/AJHE_a_00036

5. Shi P, et al. 2010. The impact of mass gatherings and holiday traveling on the course of an influenza pandemic: a computational model. BMC Public Health. 10, 778. PubMed https://doi.org/10.1186/1471-2458-10-778

6. www.wunderground.com.

7. www.marquette.edu/mucentral/registrar/ArchivedAcademicCalendars.shtml.

8. www.timeanddate.com. 
Figure 1. Weekly number tests and \% of influenza positives tests and percent positives with superimposed school holiday occurrences in Milwaukee, WI (2004-2009)

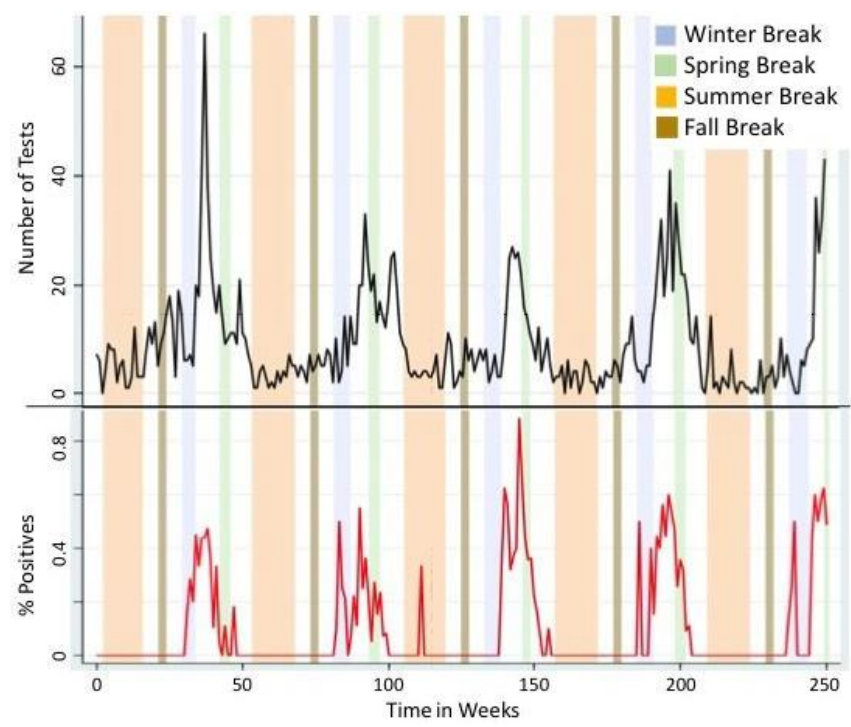

Figure 2. Weekly number of tests, positive tests, influenza A and B (panels A-D, respectively) for each study year with superimposed Winter and Spring school holiday occurrences in Milwaukee, WI. Average and standard deviation values for four outcomes and their weekly rates (in italic) for five time periods: before (5 weeks), during (2-6 weeks), and after (5 weeks) the Winter and Spring Breaks (time is shown in weeks starting September $1^{\text {st }}$ ); superscripts b and c indicate t-test significance for during-after and before-after comparisons, respectively.
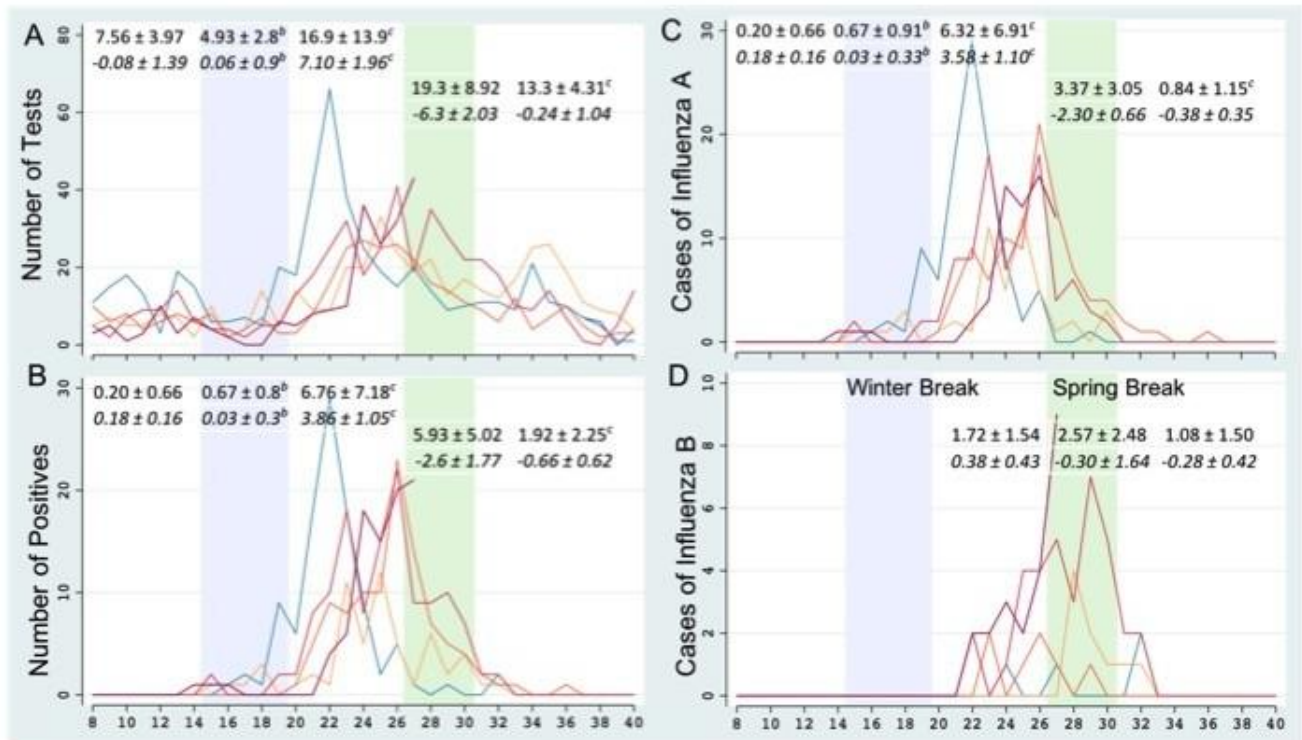

SDS Annual Conference Proceedings 2019. This is an Open Access article distributed under the terms of the Creative Commons AttributionNoncommercial 4.0 Unported License (http://creativecommons.org/licenses/by-nc/3.0/), permitting all non-commercial use, distribution, and reproduction in any medium, provided the original work is properly cited. 